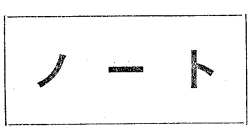

\title{
Waveform Analysis of Laser-Induced Fluorescence for Metastable Atom Density Measurement in a He Plasma
}

\author{
Hisashi SakaI, Ken Takiyama* and Toshiatsu OdA* \\ Department of Electric Engineering, Hiroshima Denki Institute of \\ Technology, Hiroshima 739-03, Japan \\ * Department of Applied Physics and Chemistry, Faculty of Engineering, \\ Hiroshima University, Higashi-Hiroshima 724, Japan
}

(Received March 16, 1994)

\begin{abstract}
酒井 恒・多幾山憲*・尾田年充*
広島電機大学電気工学科, 広島市安芸区中野 6丁目 20-1 (广7739-03)

*広島大学工学部応用理化学講座, 東広島市鏡山 1 丁目 4-1 ( 7724$)$
\end{abstract}

(1994 年 3 月 16 日受理)

\begin{abstract}
Keyword: laser-induced fluorescence, density measurement, He metastable density, glow discharge, rate equation, disalignment, polarization, atomic spectroscopy, collisional transfer
\end{abstract}

\section{Introduction}

Laser-induced fluorescence spectroscopy (LIFS) has been widely applied to measurements of the metastable atom density and other species, for instance, impurities in plasma because of its sensitivity and spatial resolution. The He metastable atom is especially important because it has been utilized for various purposes in plasma diagnostics, for example, measurements of electron density $n_{e}^{1,2)}$ and the electric field distribution in plasma sheath. $0^{3,4)}$

In LIFS, there are various kinds of atomic processes to be considered for analysis of the observed results. Let us excite the $\mathrm{He} 2^{1} \mathrm{~S}$ level to the $3^{1} \mathrm{P}$ one in a He discharge plasma by using laser with wavelength of $501.6 \mathrm{~nm}$, and observe the resonance fluorescence (RF). Collisional transfers from $3^{1} \mathrm{P}$ to $3^{1} \mathrm{D}$ are caused. Then the collision-induced fluo- rescence (CIF, $667.8 \mathrm{~nm})$ is also observed. In a weakly-ionized glow discharge plasma the transfer processes are due to the atomatom collision dominantly. In a more highly ionized plasma such as an ECR plasma, the atom-atom collision is negligible against to the electron-atom one. Tsuchida measured a spatial distribution of $n_{e}$ from the intensity ratio of $\mathrm{RF}$ to CIF in a DC plasma column $\left(n_{e}=10^{12} \mathrm{~cm}^{-3}\right)$ with an axial magnetic field of $4 \mathrm{kG}$, and then obtained the metastable population from the RF intensity distribution by solving a set of rate equations. ${ }^{2)}$

On the other hand, in intermediately-ionized plasmas, both the collisional transfers by the electron and atom become the same order of magnitude. Moreover, the effect of the alignment of atoms, that is, an anisotropic population among the sublevels of the upper one excited by the laser is one of the most essential issues to be discussed in the LIFS because the fluorescence emitted from the atoms has 
spatial anisotropy and polarization..$^{5,6)}$

In a previous paper, we applied a simplified model to determine more accurately the metastable He atom density in a hollow cathode plasma, which was measured by LIFS. ${ }^{\text {?) }}$ This method has a limitation for application to wider range of the plasma parameters: The back transfer between the upper levels was neglected. With higher electron and/or atom densities, the effect of the back transfer should be considered. This limitation will be improved by a waveform analysis of LIF signals by using a time dependent collisional radiative model.

In this paper we will develop a method to analyze the fluorescence waveforms by using rate equations. It is shown that $\mathrm{He} 2^{1} \mathrm{~S}$ metastable atom density, the electron density, transmittance of resonance fluorescence and transfer rate between the sublevels of $3^{1} \mathrm{P}$ are determined by fitting the calculated waveforms to the experimental ones. As an example, our method is applied to a hollow cathode helium plasma. The effect of the disalignment on the upper level density excited by laser is also discussed.

We describe model for waveform analysis in the next section, procedure of iterative fitting to fluorescence waveforms in the section 3 , experiment in the section 4 , and results of waveform analysis and discussion in the section 5 .

\section{Model for Waveform Analysis}

Figure 1 shows the He partial energy level diagram and collisional-radiative processes of interest. Each level is designated by number. The injected lineally polarized laser makes the $2^{1} \mathrm{~S}$ state excited only to the $m=0 \mathrm{mag}$. netic sublevel of the upper level $3^{1} \mathrm{P}$. Subsequently the $m= \pm 1$ sublevels are populated by the collisional transfer from the $m=0$ sublevel as indicated in Fig. 1(b). Then RF contains both the $\pi$ - and $\sigma$-components whose observed intensities are denoted by $i_{R} \pi$ and $i_{R} \sigma$, respectively. The $3^{1} \mathrm{D}$ level is also populated by the collisional transfer from the $3^{1} \mathrm{P}$ one. Then CIF is emitted. The $3^{1} \mathrm{D}$ level is treated as single level because no polarization in CIF was found.

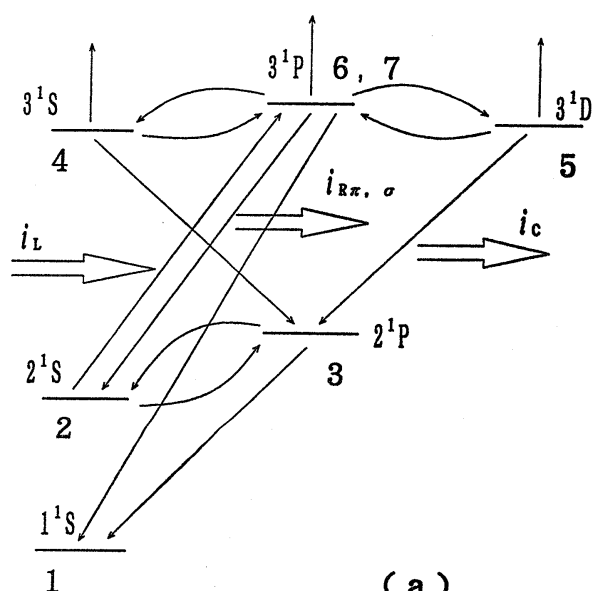

(a)

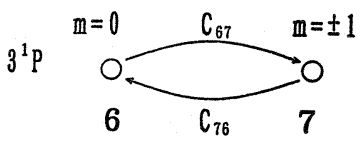

(b)

Fig. 1 Partial energy levels of HeI and transfer processes of intsrest.

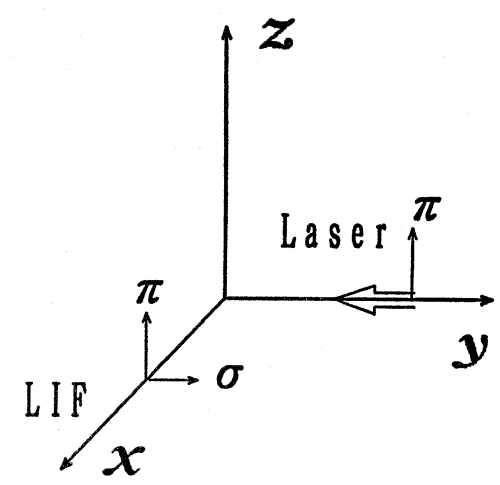

Fig. 2 Geometry of our LIFS when the He $2^{1} \mathrm{~S}$ atom is at the origin.

Figure 2 shows a geometry of our LIFS. Let the $\mathrm{He} 2^{1} \mathrm{~S}$ atom be at the origin. The laser beam comes in the opposite direction along the $y$-axis. When the observing direction (the $x$-axis) for the fluorescence is perpendicular to the laser polarization vector, the population densities of the upper levels and the observed fluorescence intensities in 
photon number unit are related in the following, ${ }^{5)}$

$$
\left(n_{6}+n_{7}\right) A_{62} \Lambda_{62} \frac{V d \Omega}{4 \pi} T_{R}=\frac{2}{3}\left\{i_{R \pi}+2 i_{R o}\right\},
$$

where $n$ 's is the population density, $A_{62}$ the spontaneous transition probability from $3^{1} \mathrm{P}$ to $2^{1} \mathrm{~S}, \Lambda_{62}$ the optical escape factor, $V$ the observing volume, $d \Omega$ the solid angle and $T_{R}$ the transmittance of RF through the plasma. From eq. (1) we obtain

$$
i_{R \pi}=\frac{3}{2} n_{6} A_{62} \Lambda_{62} \frac{V d \Omega}{4 \pi} T_{R},
$$

and

$$
i_{R \sigma}=\frac{3}{4} n_{7} A_{72} \Lambda_{72} \frac{V d \Omega}{4 \pi} T_{R},
$$

where $A_{62}$ equals $A_{72}$. On the other hand, CIF is assumed to be free from polarization and reabsorption, so that the intensity $i_{C}$ is given by

$$
i_{C}=n_{5} A_{53} \frac{V d \Omega}{4 \pi} .
$$

The rate equation for the population density is given by

$$
\begin{aligned}
\frac{d n_{i}}{d t}= & \sum_{j>i} \Lambda_{j i} A_{j i} h_{j}+\sum_{k \neq i} C_{k i} n_{k}-n_{i}\left(\sum_{m<i} \Lambda_{i m} A_{i m}\right. \\
& \left.+\sum_{n \neq i} C_{i n}+R_{i}\right)+\left(\delta_{i 6}-\delta_{2 i}\right) i_{L}\left(n_{2} B_{28}\right. \\
& \left.-n_{6} B_{62}\right), \quad i=2, \ldots, 7
\end{aligned}
$$

where $A$ 's and $B$ 's are the Einstein's coefficients, ${ }^{8)} C$ 's the transfer rates between the excited levels due to both the electronic collision $^{9)}$ and atomic collision ${ }^{10)} R$ 's the ionization rates, ${ }^{9,10)} \delta$ the Kronecker's delta and $i_{L}$ the laser spectral power density. The coefficients $C_{67}$ and $C_{76}$ mean the transfer rate from the $m=0$ to $m= \pm 1$ sublevels and the corresponding one of the inverse process, respectively.

Since no data of $C_{67}$ and $C_{78}$ are available, these values have to be estimated from fitting the calculated time behaviors to the experimental ones of the $\pi$ - and $\sigma$-light as described later. The values of $n_{e}, n_{2}(t=0)$, $\Lambda_{62}\left(=\Lambda_{72}\right)$ and $T_{R}$ are also determined by iterative fitting of the calculated waveforms to the observed ones of RF and CIF.
By solving numerically the rate equation (5) with the Runge Kutta method, we obtain temporal behaviors of the population densities, and then get the corresponding curves of $i_{R \pi}, i_{R \sigma}$ and $i_{C}$ by using eqs. (2)-(4) In order to perform fitting with the experimental waveforms of RF and CIF we make convolution product of the time response function of our spectroscopic system on the calculated curves of $i_{R \pi}, i_{R \sigma}$ and $i_{c}$.

\section{Procedure of Iterative Fitting to Fluorescence Waveforms}

First of all, we assume the starting values of the necessary parameters for our iterative calculation. The values of $n_{e}$ and $T_{e}$ in the negative glow region are determined by probe measurement described in the section 4. The value of $n_{e}$ is also determined by iterative fitting as described later. The starting value of $n_{2}(t=0)$ is presumed from the total absolute intensities of RF and CIF because we assume that about half of the $2^{1} \mathrm{~S}$ population density is excited to the $3^{1} \mathrm{P}$ level. Each escape factor is estimated from the lower level density and the laser beam size using Table 3 in Ref. 11. We assume the starting values of the other necessary parameters to be $C_{67}\left(=2 C_{76}\right)=0$ and $T_{R}=1$.

The following procedures (1)-(5) are iterated until the parameter values converge.

(1) The decay curves of $i_{R \pi}$ and $i_{R \sigma}$, and also of $i_{C}$, are largely dependent on $n_{e}$. By changing the numerical value of $n_{e}$ to calculate the $C$ and $R$ rates, we solve repeatedly eq. (5), and obtain the best fit curve of $i_{C}$ to the corresponding experimental one in its decay period. This procedure determines $n_{e}$.

(2) The peak region of the $i_{R \pi}$ and $i_{R \sigma}$ curves so far calculated are not yet fitted to the experimental one. We have to repeat solving eq. (5) by changing the value of $C_{67}$ $\left(=2 C_{76}\right)$ with all the other coefficients kept constant to get the best fit curves there.

(3) In order to determine the value of $n_{2}$ at $t=0$, at the time, just before the exciting laser is on, the calculated total intensity of the $667.8 \mathrm{~nm}$ fluorescence $I_{C C}$, which is obtained from the temporal integration of $i_{C}$, is compared with the observed absolute total 
intensity $I_{C e}$ because it is proportional to $n_{2}$ at $t=0$. The ratio $k=I_{C C} / I_{C e}$ is then multiplied to the starting value of $n_{2}(t=0)$ to obtain the iterative value of $n_{2}(t=0)$.

(4) Using the obtained values of $n_{2}(t=0)$ the escape factors, $\Lambda_{62}$ and $\Lambda_{72}$ are calculated again.

(5) We determine $T_{R}$ by normalizing the calculated curves of $i_{R \pi}$ and $i_{R \sigma}$ to the absolutely calibrated experimental curves of RF.

\section{Experiment}

Experimental apparatus is similar to that described in Ref. 3. A plane parallel hollow cathode consists of a pair of disc electrodes with a diameter of $4 \mathrm{~cm}$ and a separation of $11 \mathrm{~mm}$ and is placed normally to the $z$ axis whose origin corresponds to the center of the gap of electrodes. The hollow-cathode plasma (the negative glow plasma) was produced between the gap by the DC discharge with the current of $40 \mathrm{~mA}$ and He gas pressure of 0.7 Torr $(90 \mathrm{~Pa})$. The electron temperature $T_{e}$ and the electron density $n_{e}$ at the center of the negative glow were measured by a Langmuir probe to be $1.3 \mathrm{eV}$ and $(2.0 \pm 0.5) \times 10^{11} \mathrm{~cm}^{-3}$, respectively.

As shown in Fig. 2, a YAG-laser-pumped dye laser light, polarized linearly parallel to the $z$-axis (quantization axis), was introduced into the plasma along the $y$-axis $(\pi$-light excitation). The pulse energy was about $10 \mu$ $\mathrm{J}$, the pulse width (FWHM) $3 \mathrm{~ns}$, the spectral width $4 \mathrm{pm}$, and the beam cross section 4.0 $\times 1.0 \mathrm{~mm}^{2}$. The resonance fluorescence $(\mathrm{RF}$ : $501.6 \mathrm{~nm}$ ) and the collision induced one (CIF: $667.8 \mathrm{~nm})$ were observed along the $x$-axis. The $\pi$ - and $\sigma$-components were separated by using a sheet polarizer (Polaroid HN32) and observed with a $25 \mathrm{~cm}$ monochromator using a gated photomultipiler (Hamamatsu R928).

The laser-pulse waveform was also measured by a biplaner phototube (Hamamatsu R1193). The time response function of the our spectroscopic system was approximated with double Gaussian distribution with the half width at half maximum of $1.5 \mathrm{~ns}$ on the rising side and $5.6 \mathrm{~ns}$ on the falling side. This distribution was estimated from the difference between the laser waveforms detected

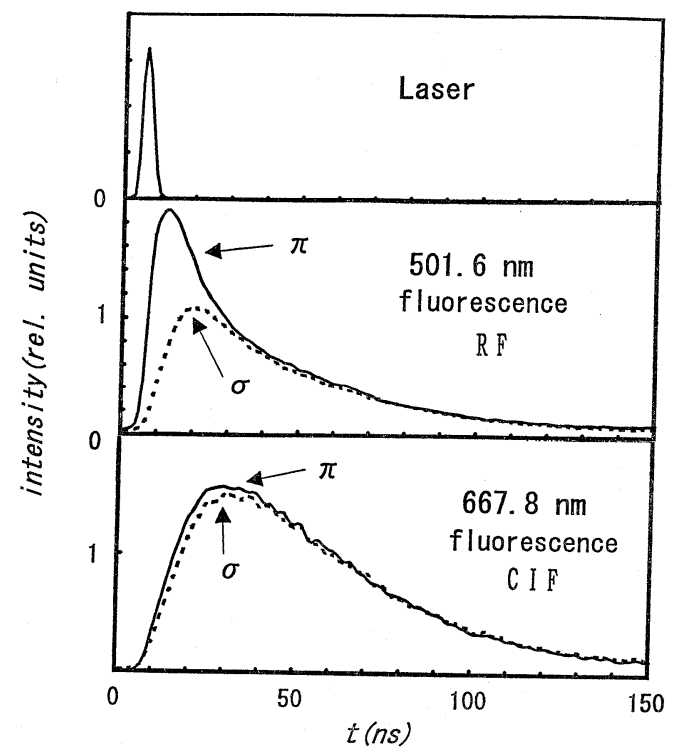

Fig. 3 Observed temporal behaviors of dye laser, resonance $501.6 \mathrm{~nm}$ fluorescence and collision-induced $667.8 \mathrm{~nm}$ fluorescence where solid lines and broken lines show the $\pi$ and $\sigma$-components, respectively.

by the biplanner and by the photomultiplier used in the spectroscopic system.

Figure 3 shows the experimental waveforms of the laser, $\mathrm{RF}(501.6 \mathrm{~nm})$ and $\mathrm{CIF}$ $667.8 \mathrm{~nm})$. In the initial stage of its temporal behavior RF almost consists of only $\pi$-light. Because the laser is linearly polarized, only $m=0$ sublevel of $3^{1} \mathrm{P}$ is populated by $\pi$-light excitation. And $\sigma$-light becomes the same intensity as $\pi$-light at around $25 \mathrm{~ns}$; the disalignment takes place. This is probably due to the collisional transfer between the magnetic sublevels in the $3^{1} \mathrm{P}$ level. The polarization of CIF was not observed even at the early stage within the experimental error. The possible reasons are that the alignment may not be produced among the sublevels of the $3^{1} \mathrm{D}$ level by the collisional transfer and the emission line from the $3^{1} \mathrm{D}$ to $2^{1} \mathrm{P}$ level is hardly polarized.

\section{Results of Waveform Analysis and Discussion}

Calculated temporal behaviors of the fluorescence are shown with the solid lines in 


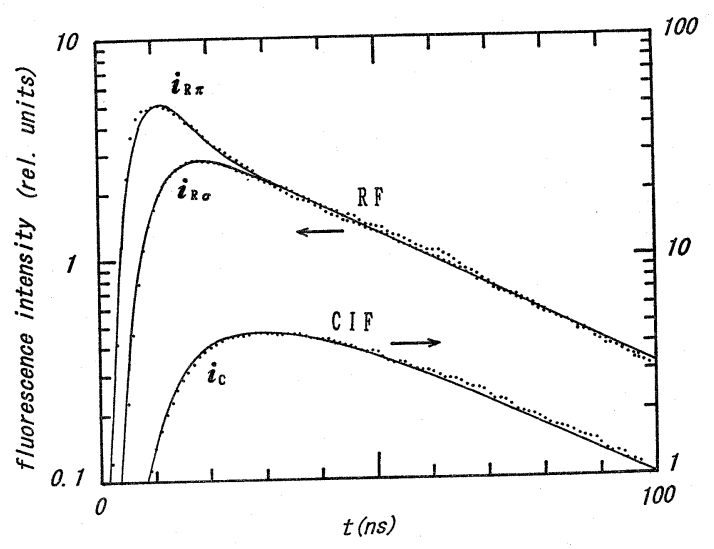

Fig. 4 Calculated waveforms (solid lines) fitted to the experimental ones (dotted lines). The obtained plasma parameters are as follows: $T_{R}=0.85, n_{2}(t=0)=1.0 \times 10^{11} \mathrm{~cm}^{-3}$, $C_{67}=2 C_{76}=1.9 \times 10^{8} \mathrm{~s}^{-1}$ and $n_{e}=2.0 \times 10^{11}$ $\mathrm{cm}^{-3}$, which are determined according to the procedure described in the section 3 .

Fig. 4. The scale of $i_{R \pi}$ and $i_{R \sigma}(501.6 \mathrm{~nm})$ is expressed by the ordinate in the left hand side and that of $i_{c}(667.8 \mathrm{~nm})$ in the right one. These are in good agreement with the experimental results (dotted lines). The determined parameters were $T_{R}=0.85 \pm 0.03, n_{2}$ $(t=0)=(1.0 \pm 0.1) \times 10^{11} \mathrm{~cm}^{-3}, C_{67}=2 C_{76}=(1.9 \pm$ $0.2) \times 10^{8} \mathrm{~s}^{-1}$ and $n_{e}=(2.0 \pm 0.2) \times 10^{11} \mathrm{~cm}^{-3}$.

To show reliability of our fittings made as in Fig. 4, we also calculated temporal behaviors of the longitudinal alignment $\left(L_{A}\right)$ defined by $L_{A}=\left(i_{R \pi}-i_{R \sigma}\right) /\left(i_{R \pi}+2 i_{R \sigma}\right)^{5)}$ by using $i_{R \pi}$ and $i_{R \sigma}$, which are obtained in our calculation, and fitted it to the corresponding experimental one. The reason is that $L_{A}$ is much more sensitive to misfit of our calculation to the experimental result than the culculated curves of $i_{R \pi}$ and $i_{R \sigma}$. Figure 5 shows that the calculated $L_{A}$ denoted by solid line is well fitted with the experimental one denoted by open circles. This indicates that our model and procedure for the waveform analysis of the laser-induced fluorescence are quite reasonable to determine the metastable level population and collisional transfer rate. Small discrepancy found in the early stage corresponds to the small difference between the calculated and experimental curves of $i_{R \pi}$ and $i_{R \sigma}$ in the rise time shown in Fig. 4. This is probably due to the approximation

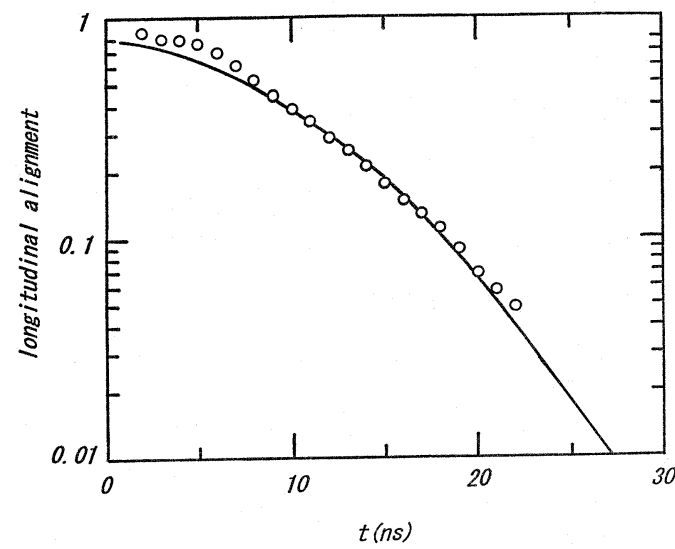

Fig. 5 Time variations of longitudinal alignment where solid line and open circles show the theoretical and experimental results, respectively.

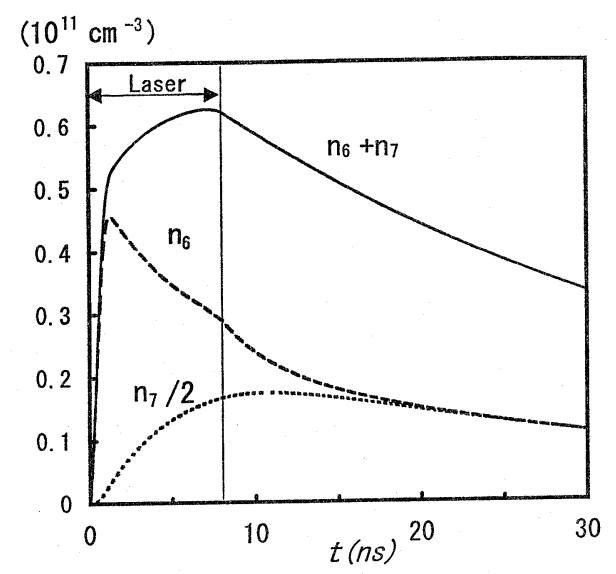

Fig. 6 Calculated temporal behaviors of $n_{6}, n_{7} / 2$ and $n_{6}+n_{7}$.

of the time response function of observing apparatus.

Thus uncertainty of the obtained value of $C_{67}\left(=2 C_{76}\right)$ is inferred to be mainly due to the experimental error in the measurement of $i_{R \pi}$ and $i_{R \sigma}$. The obtained $C_{67}$ is one order of magnitude larger than the transfer rate, $C_{65}$. The reciprocal of $C_{67}$ is $5 \mathrm{~ns}$. This value is comparable to the laser pulse duration, so that the transfer between the magnetic sublevels takes place even during the laser excitation.

Our waveform curves shown in Figs. 4 and 5 include the time response of the spectroscopic system used, as already described in 
the section 2. To directly show temporal behavior of the collisionl transfer between the sublevels of $3^{1} \mathrm{P}$ level, we demonstrate time evolutions of the calculated population densities $n_{6}(m=0)$ and $n_{7}(m= \pm 1)$ of the sublevels in Fig. 6. The injected laser immediately makes distribution between $n_{6}$ and $n_{2}$ to be nearly $1: 1$, while $n_{7}$ starts to increase due to the collisional transfer, that is, the disalignment begins. After a sharp rise of $n_{6}$ in the very early stage $(0 \leq t \leq 1 \mathrm{~ns})$, the total population $n_{6}+n_{7}$ still keeps increasing during the laser irradiation. Such a rather complicated change of the population densities due to the selective excitation by laser suggests that effect of the disalignment should be taken into account for LIFS.

In conclusion, we have demontrated that in LIFS our waveform analysis of the laserinduced fluorescence is useful for determination of the metastable level population in a plasma, where we have to consider the polarization of the fluorescence as well as the absorption of the resonance fluorescence in the plasma.

\section{Acknowledgements}

We would like to thank Prof. U. Furukane for his valuable discussions. This work was supported in part by a Grant-in-Aid for Scientific Research from the Ministry of Education, Science and Culture.

\section{References}

1) K. Tsuchida, S. Miyake, K. Kadota, and J. Fujita: Plasma Phys. 25, 991 (1983).

2) K. Tsuchida: Jpn. J. Appl. Phys. 23, 338 (1984).

3) H. Sakai, K. Takiyama, M. Kimura, M. Yamasaki, T. Oda, and K. Kawasaki: J. Nucl. Mater. 196-198, 1135 (1992).

4) H. Sakai, K. Takiyama, T. Fujita, M. Tsuji, T. Oda, and K. Kawasaki: J. Spectrosc. Soc. Japan 40, 275 (1991).

5) T. Fujimoto, C. Goto, Y. Uetani, and K. Fukuda: Jpn. J. Appl. Phys. 24, 875 (1985); A. Hirabayashi, Y. Nambu, and T. Fujimoto: ibid. 25, 1563 (1986).

6) A. Hirabayashi, Y. Nambu, M. Hasuo, and T. Fujimoto: Phys. Rev. A37, 83 (1988).

7) K. Takiyama, H. Sakai, M. Yamasaki, and T. Oda: Jpn. J. Appl. Phys. (1994) to be published.

8) W. L. Wiese, M. W. Smith, and B. M. Glennon: Natl. Stand. Ref. Data Ser. 1 (1966).

9) T. Fujimoto: J. Quant. Spectrosc. Radiat. Transfer 21, 439 (1979).

10) B. Dubreuil and A. Catherinot: Phys. Rev. A21, 188 (1980).

11) M. Otsuka, R. Ikee, and K. Ishii: J. Quant. Spectrosc. Radiat. Transfer 21, 41 (1979). 